\title{
Reply to the editorial comment from Van der Wall concerning 'The right ventricle: always normal in normal subjects?'
}

\author{
S. Quick · R.H. Strasser · U. Speiser
}

Published online: 8 April 2015

(C) The Author(s) 2015. This article is published with open access at Springerlink.com

To the Editor,

We read the comment referring to the challenges in imaging the right ventricle with great interest [1]. The difficulties in distinguishing wall motion abnormalities in healthy subjects from those in diseased patients are very well summarised. The author cites the article by Doesch et al. [2] presenting 20 healthy subjects with CMR-derived measurement of the tricuspid annular plane systolic excursion (TAPSE) with a reference point outside the ventricle (TAPSEout), which might be used for screening right ventricular motion.

We could demonstrate TAPSE in cardiac magnetic resonance (CMR), being a fast and easily obtainable parameter correlating well to volumetric quantification of right ventricular ejection fraction (RVEF), with low interobserver and intraobserver variables. Therefore, we investigated 76 patients (age: $58 \pm 17$ years) with mean RVEF of $42 \pm 14 \%$, assessed by the standardised slice-summation method. CMR-TAPSE was determined to be $19 \pm 6 \mathrm{~mm}$ and correlated well in linear regression analysis with volumetric RVEF $(r=0.72, p<0.001)$. However, we could also show that CMR-TAPSE is not only a fast tool in healthy subjects, but it also discriminates well between patients with impaired and normal RVEF. Multiplying CMR-TAPSE measurements by 2.5 leads to values close to the RVEF by volumetry. Furthermore, CMR-TAPSE correlates well with TAPSE determined using transthoracic echocardiography [3].

Funding None.

Conflict of interest None declared.

Open Access This article is distributed under the terms of the Creative Commons Attribution License which permits any use, distribution, and reproduction in any medium, provided the original author(s) and the source are credited.

\section{References}

1. Van der Wall EE. The right ventricle: always normal in normal subjects. Neth Heart J. 2015;23:62-3.

2. Doesch C, Zompolou C, Streitner F, et al. CMR-derived TAPSE measurement: a semi-quantitative method of right ventricular function assessment in patients with hypertrophic cardiomyopathy. Neth Heart J. 2014;22:557-64.

3. Speiser U, Hirschberger M, Pilz G, et al. Tricuspid annular plane systolic excursion assessed using MRI for semi-quantification of right ventricular ejection fraction. Br J Radiol. 2012;85:e716-21.
U. Speiser $(\bowtie) \cdot$ S. Quick $\cdot$ R.H. Strasser

Klinik für Innere Medizin und Kardiologie, Herzzentrum

Dresden Universitätsklinik, Technische Universität Dresden,

Fetscherstraße 76,

Dresden 01307, Germany

e-mail: uwe.speiser@mailbox.tu-dresden.de 
